\title{
ON UNIFORMLY CLOSE-TO-CONVEX FUNCTIONS AND UNIFORMLY QUASICONVEX FUNCTIONS
}

\section{K. G. SUBRAMANIAN, T. V. SUDHARSAN, and HERB SILVERMAN}

\author{
Received 30 October 2002
}

\begin{abstract}
Two new subclasses of uniformly convex and uniformly close-to-convex functions are introduced. We obtain inclusion relationships and coefficient bounds for these classes.
\end{abstract}

2000 Mathematics Subject Classification: 30C45.

1. The class $\operatorname{UCC}(\alpha)$. Denote by $S$ the family consisting of functions

$$
f(z)=z+\sum_{n=2}^{\infty} a_{n} z^{n}
$$

that are analytic and univalent in $\Delta=\{z:|z|<1\}$ and by $C, S^{*}$, and $K$ the subfamilies of functions that are, respectively, convex, starlike, and close to convex in $\Delta$. Noor and Thomas [7] introduced the class of functions known as quasiconvex functions. A normalized function of the form (1.1) is said to be quasiconvex in $\Delta$ if there exists a convex function $g$ with $g(0)=0, g^{\prime}(0)=1$ such that for $z \in \Delta$,

$$
\operatorname{Re} \frac{\left(z f^{\prime}(z)\right)^{\prime}}{g^{\prime}(z)}>0
$$

Let $Q$ denote the class of quasiconvex functions defined in $\Delta$. It was shown that $Q \prec K$, where $\prec$ denotes subordination, so that every quasiconvex function is close to convex. Goodman [2, 3] introduced the classes UCV and UST of uniformly convex and uniformly starlike functions. In [10], Rønning defined the class $\operatorname{UCV}(\alpha),-1 \leq \alpha<1$, consisting of functions of the form (1.1) satisfying

$$
\operatorname{Re}\left(1+\frac{z f^{\prime \prime}(z)}{f^{\prime}(z)}\right)-\alpha \geq\left|\frac{z f^{\prime \prime}(z)}{f^{\prime}(z)}\right|, \quad z \in \Delta
$$

Geometrically, $\operatorname{UCV}(\alpha)$ is the family of functions $f$ for which $1+z f^{\prime \prime}(z) / f^{\prime}(z)$ takes values that lie inside the parabola $\Omega=\{\omega: \operatorname{Re}(\omega-\alpha)>|\omega-1|\}$, which is symmetric about the real axis and whose vertex is $w=(1+\alpha) / 2$. 
Since the function

$$
q_{\alpha}(z)=1+\frac{2(1-\alpha)}{\pi^{2}}\left(\log \frac{1+\sqrt{z}}{1-\sqrt{z}}\right)^{2}
$$

maps $\Delta$ onto this parabolic region, $f \in \mathrm{UCV}(\alpha)$ if and only if

$$
1+\frac{z f^{\prime \prime}(z)}{f^{\prime}(z)} \prec q_{\alpha}(z)
$$

Rønning [10] also defined the family $S_{p}(\alpha)$ consisting of functions $z f^{\prime}(z)$ when $f$ is in $\operatorname{UCV}(\alpha)$. In particular, $f$ is in $S_{p}(\alpha)$ if and only if $z f^{\prime}(z) / f(z) \prec$ $q_{\alpha}(z)$.

Note for $g(z)=z f^{\prime}(z) / f(z)$ that $g(z)+z g^{\prime}(z) / g(z)=1+z f^{\prime \prime}(z) / f^{\prime}(z)$, and hence a result of Miller and Mocanu [6] shows that $\operatorname{UCV}(\alpha) \subset S_{p}(\alpha)$.

Kumar and Ramesha [4] investigated the class UCC of uniformly close-toconvex functions consisting of normalized functions of the form (1.1) satisfying $f^{\prime}(z) / g^{\prime}(z) \prec q_{0}(z)$, where $g(z) \in C$ and $q_{0}(z)$ is given by (1.4) for $\alpha=0$.

More generally, we give the following definition.

DEFINITION 1.1. A function $f$ is said to be uniformly close to convex of order $\alpha,-1 \leq \alpha<1$, denoted by $\operatorname{UCC}(\alpha)$, if $f^{\prime}(z) / g^{\prime}(z) \prec q_{\alpha}(z)$, where $q_{\alpha}(z)$ is as defined by (1.4) and $g(z)$ is convex.

Since $\operatorname{Re} q_{\alpha}(z)>0$, we see that $\operatorname{UCC}(\alpha)$ is a subclass of $K$. To see that UCC $(\alpha)$ also contains the family $S_{p}(\alpha)$, we note for $f \in S_{p}(\alpha) \subset S^{*}$ that $f(z)=z g^{\prime}(z)$ for some $g \in C$. Hence, $z f^{\prime}(z) / f(z)=f^{\prime}(z) / g^{\prime}(z) \prec q_{\alpha}(z)$.

We have thus proved the following inclusion chain.

THEOREM 1.2. For $-1 \leq \alpha<1, \mathrm{UCV}(\alpha) \prec S_{p}(\alpha) \prec \mathrm{UCC}(\alpha) \prec K$.

We next give a sufficient condition for a function to be in $\operatorname{UCC}(\alpha)$.

THEOREM 1.3. If $\sum_{n=2}^{\infty} n\left|a_{n}\right| \leq(1-\alpha) / 2$, then $f(z)=z+\sum_{n=2}^{\infty} a_{n} z^{n}$ is in $\operatorname{UCC}(\alpha),-1 \leq \alpha<1$.

Proof. Setting $g(z)=z$, we have $f^{\prime}(z) / g^{\prime}(z)=f^{\prime}(z)=1+\sum_{n=2}^{\infty} n a_{n} z^{n-1}$, so that for $z \in \Delta$,

$$
\left|\frac{f^{\prime}(z)}{g^{\prime}(z)}-1\right|<\sum_{n=2}^{\infty} n\left|a_{n}\right| \leq 1-\sum_{n=2}^{\infty} n\left|a_{n}\right|-\alpha \leq \operatorname{Re} f^{\prime}(z)-\alpha .
$$

Thus $f^{\prime}(z) / g^{\prime}(z)$ lies in the parabolic region $\Omega=\{\omega:|\omega-1|<\operatorname{Re}(\omega-\alpha)\}$. That is, $f^{\prime}(z) / g^{\prime}(z) \prec q_{\alpha}(z)$, where $q_{\alpha}(z)$ is as defined by (1.4). 
2. A convolution relation. We now prove a convolution result for the family $\operatorname{UCC}(\alpha)$. But first we need the following lemma.

LEMMA 2.1 (see [8]). Let $\phi(z) \in C, \psi \in S^{*}$. If $F(z)$ is analytic and $\operatorname{Re}\{F(z)\}>$ $\alpha,-1 \leq \alpha<1$, then

$$
\operatorname{Re}\left\{\frac{\phi * F \psi}{\phi * \psi}\right\}>\alpha, \quad z \in \Delta
$$

The above result was proved in [11] for the case $\alpha=0$.

THEOREM 2.2. If $f \in \operatorname{UCC}(\alpha)$, then to each $g \in S^{*}$, an $h \in S^{*}$ may be associated for which $\operatorname{Re}(f * g) / h>(1+\alpha) / 2, z \in \Delta$.

Proof. If $f \in \operatorname{UCC}(\alpha)$, then $f^{\prime}(z) / g_{1}^{\prime}(z) \prec q_{\alpha}(z)$, where $g_{1}(z) \in C$ and $q_{\alpha}(z)$ is defined by (1.4). Hence, $\operatorname{Re}\left(f^{\prime}(z) / g_{1}^{\prime}(z)\right)>(1+\alpha) / 2$. Therefore, we can find an $\psi \in S^{*}$ for which

$$
\operatorname{Re} \frac{z f^{\prime}(z)}{\psi(z)}>\frac{1+\alpha}{2}
$$

Set $F(z)=z f^{\prime}(z) / \psi(z)$. Then, for $g \in S^{*}$, there corresponds a $\phi \in C$ such that $z \phi^{\prime}=g$. Also $f * g=z f^{\prime} * \phi=\phi * F \psi$ and $h=\phi * \psi \in S^{*}$. By Lemma 2.1,

$$
\operatorname{Re} \frac{\Phi * F \Psi}{\Phi * \Psi}=\operatorname{Re} \frac{f * g}{h}>\frac{1+\alpha}{2}
$$

and this proves the result.

3. Coefficient estimates. We need the following result by Rogosinski [9] to obtain coefficient bounds for the class $\operatorname{UCC}(\alpha)$.

LEMMA 3.1. Let $h(z)=1+\sum_{k=1}^{\infty} c_{k} z^{k}$ be subordinate to $H(z)=1+\sum_{k=1}^{\infty} C_{k} z^{k}$. If $H(z)$ is univalent in $\Delta$ and $H(\Delta)$ is convex, then $\left|c_{n}\right| \leq\left|C_{1}\right|$.

THEOREM 3.2. If $f(z)=z+\sum_{n=2}^{\infty} a_{n} z^{n} \in \operatorname{UCC}(\alpha)$, then

$$
\left|a_{n}\right| \leq(n-1) c+1, \quad n \geq 2,
$$

where $c=4(1-\alpha) / \pi^{2}$.

Proof. Set

$$
\Phi(z)=\frac{f^{\prime}(z)}{g^{\prime}(z)}=1+\sum_{k=1}^{\infty} c_{k} z^{k}
$$

so that $\Phi(z) \prec q_{\alpha}(z)$, where $q_{\alpha}(z)$ is defined in (1.4). 
Since $q_{\alpha}(z)$ is univalent and maps $\Delta$ onto a convex region, we may apply Lemma 3.1.

Now

$$
q_{\alpha}(z)=1+\frac{8(1-\alpha)}{\pi^{2}} z+\cdots, \quad \text { so that }\left|c_{n}\right| \leq \frac{8(1-\alpha)}{\pi^{2}}
$$

With $g(z)=z+\sum_{k=2}^{\infty} b_{k} z^{k}$, we compare the coefficients of $z^{n}$ for the expansion of $\phi(z)$ to obtain

$$
(n+1)\left|a_{n+1}\right|=c_{n}+\sum_{k=1}^{n-1}(k+1) b_{k+1} c_{n-k}+(n+1) b_{n+1} .
$$

Since $g(z)$ is convex, it is well known that $\left|b_{n}\right| \leq 1, n=1,2, \ldots$ From (3.4), we get

$$
(n+1)\left|a_{n+1}\right| \leq c n(n+1)+(n+1),
$$

and the proof is complete.

4. The class $\operatorname{UQC}(\alpha)$. We now introduce a natural analogue to the class $\mathrm{UCV}(\alpha)$ in terms of Alexander's result on convex functions [1, page 43].

DEFINITION 4.1. A normalized function of the form (1.1) is said to be uniformly quasiconvex of order $\alpha,-1 \leq \alpha<1$, in $\Delta$, denoted by $\operatorname{UQC}(\alpha)$, if there exists a convex function $g(z)$ with $g(0)=0, g^{\prime}(0)=1$, such that

$$
\frac{\left(z f^{\prime}(z)\right)^{\prime}}{g^{\prime}(z)} \prec q_{\alpha}(z),
$$

where $q_{\alpha}(z)$ is as defined by (1.4).

REMARK 4.2. (1) By setting $f(z)=g(z)$, we see that $\operatorname{UCV}(\alpha) \subset \operatorname{UQC}(\alpha)$.

(2) We see that $f \in \operatorname{UQC}(\alpha)$ if and only if $z f^{\prime} \in \operatorname{UCC}(\alpha)$.

In view of the above remark, we obtain from Theorem 1.3 a sufficient coefficient bound for inclusion in the family $\operatorname{UQC}(\alpha)$.

THEOREM 4.3. If $\sum_{n=2}^{\infty} n^{2}\left|a_{n}\right| \leq(1-\alpha) / 2$, then $f(z)=z+\sum_{n=2}^{\infty} a_{n} z^{n} \in$ $\operatorname{UQC}(\alpha)$.

We next prove a theorem which shows that every function in $\operatorname{UQC}(\alpha)$ is close to convex and hence univalent. We need a result due to Miller and Mocanu [5].

LEMMA 4.4. Let $M(z)$ and $N(z)$ be regular in $\Delta$ with $M(z)=N(z)=0$ and let $\alpha$ be real. If $N(z)$ maps $\Delta$ onto a possibly many-sheeted region which is starlike with respect to the origin, then for $z \in \Delta$,

$$
\operatorname{Re} \frac{M^{\prime}(z)}{N^{\prime}(z)}>\alpha \Rightarrow \operatorname{Re} \frac{M(z)}{N(z)}>\alpha
$$


THEOREM 4.5. If $F(z) \in \operatorname{UQC}(\alpha)$, then $F(z) \in K$ and hence it is univalent in $\Delta$.

Proof. Since

$$
\frac{\left(z f^{\prime}(z)\right)^{\prime}}{g^{\prime}(z)} \prec q_{\alpha}(z) \Longrightarrow \operatorname{Re}\left\{\frac{\left(z f^{\prime}(z)\right)^{\prime}}{g^{\prime}(z)}\right\}>\frac{1+\alpha}{2},
$$

an application of Lemma 4.4, with $M(z)=z f^{\prime}(z), N(z)=g(z)$, proves the result.

THEOREM 4.6. If $f(z) \in \operatorname{UQC}(\alpha)$, then $H(z)=\int_{0}^{z}\left(t f^{\prime}(t)\right)^{\prime} d t$ is in $\operatorname{UCC}(\alpha)$.

Proof. If $f(z) \in \operatorname{UQC}(\alpha)$, then there exists a function $g(z) \in C$ such that $\left(z f^{\prime}(z)\right)^{\prime} / g^{\prime}(z) \prec q_{\alpha}(z)$, where $q_{\alpha}(z)$ is as given by (1.4). The result now follows on observing that $H^{\prime}(z)=\left(z f^{\prime}(z)\right)^{\prime}$.

We close with coefficient estimates for the class $\operatorname{UQC}(\alpha)$.

THEOREM 4.7. If $f(z)=z+\sum_{n=2}^{\infty} a_{n} z^{n} \in \operatorname{UQC}(\alpha)$, then

$$
\left|a_{n}\right| \leq \frac{(n-1) c+1}{n}, \quad n \geq 2,
$$

where $c=4(1-\alpha) / \pi^{2}$.

Proof. Proceeding on the same lines as in the proof of Theorem 3.2, we obtain the result.

REMARK 4.8. When $\alpha=0$, UQC $(0)=Q$ [6] and we see that the bounds are lower than the corresponding bounds for $Q$ in [6].

\section{REFERENCES}

[1] P. L. Duren, Univalent Functions, Grundlehren der Mathematischen Wissenschaften, vol. 259, Springer-Verlag, New York, 1983.

[2] A. W. Goodman, On uniformly convex functions, Ann. Polon. Math. 56 (1991), no. 1, 87-92.

[3] _ On uniformly starlike functions, J. Math. Anal. Appl. 155 (1991), no. 2, 364-370.

[4] S. Kumar and C. Ramesha, Subordination properties of uniformly convex and uniformly close to convex functions, J. Ramanujan Math. Soc. 9 (1994), no. 2, 203-214.

[5] S. S. Miller and P. T. Mocanu, Second-order differential inequalities in the complex plane, J. Math. Anal. Appl. 65 (1978), no. 2, 289-305.

[6] __ On some classes of first-order differential subordinations, Michigan Math. J. 32 (1985), no. 2, 185-195.

[7] K. I. Noor and D. K. Thomas, Quasi-convex univalent functions, Int. J. Math. Math. Sci. 3 (1980), no. 2, 255-266.

[8] S. Owa, On convolution theorems, Proc. Japan Acad. Ser. A Math. Sci. 65 (1989), no. $6,161-162$.

[9] W. Rogosinski, On the coefficients of subordinate functions, Proc. London Math. Soc. (2) 48 (1943), 48-82. 
[10] F. Rønning, On starlike functions associated with parabolic regions, Ann. Univ. Mariae Curie-Skłodowska Sect. A 45 (1991), 117-122.

[11] St. Ruscheweyh and T. Sheil-Small, Hadamard products of Schlicht functions and the Pólya-Schoenberg conjecture, Comment. Math. Helv. 48 (1973), 119135.

K. G. Subramanian: Department of Mathematics, Madras Christian College, Tambaram, Chennai 600 059, India

T. V. Sudharsan: Department of Mathematics, South India Vaniar Educational Trust (SIVET) College, Gowrivakkam, Chennai 601 302, India

Herb Silverman: Department of Mathematics, University of Charleston, Charleston, SC 29424, USA

E-mail address: si 1vermanh@cofc.edu 


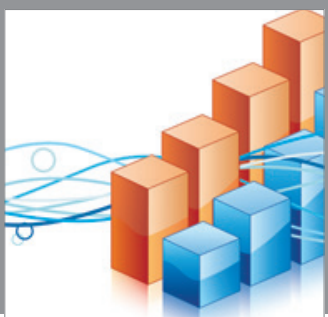

Advances in

Operations Research

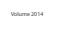

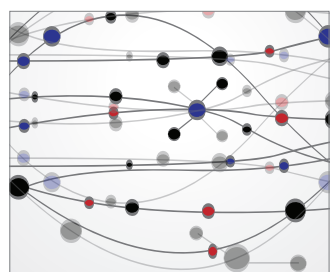

\section{The Scientific} World Journal


International Journal of

Mathematics and

Mathematical

Sciences
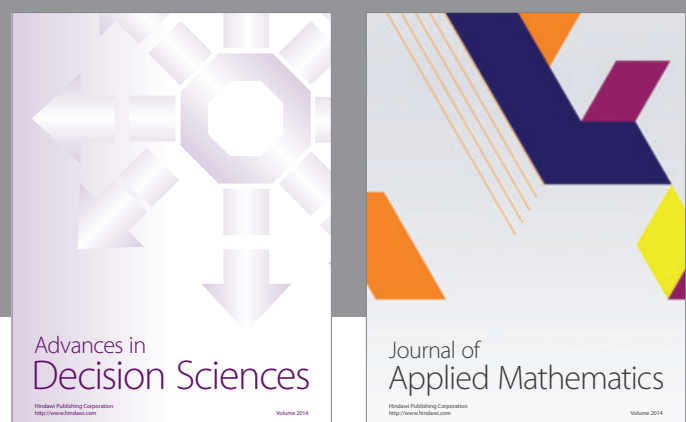

Journal of

Applied Mathematics
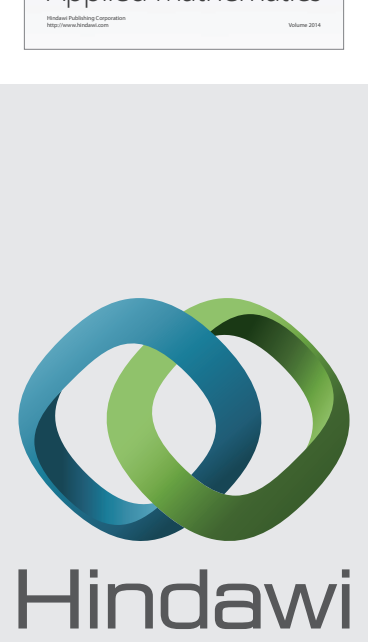

Submit your manuscripts at http://www.hindawi.com
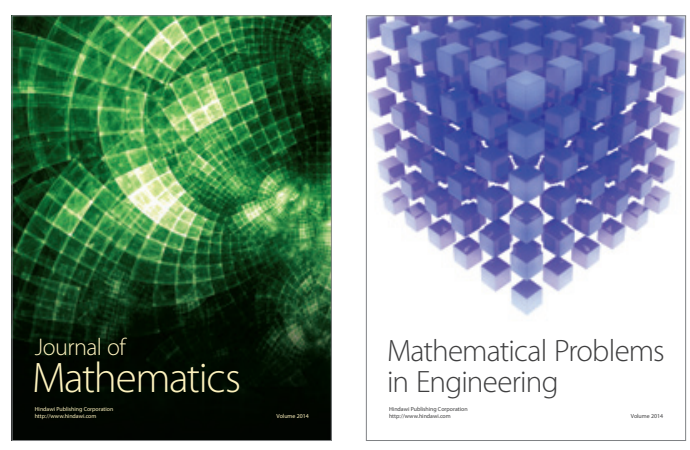

Mathematical Problems in Engineering
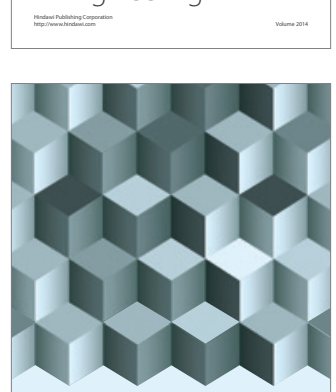

Journal of

Function Spaces
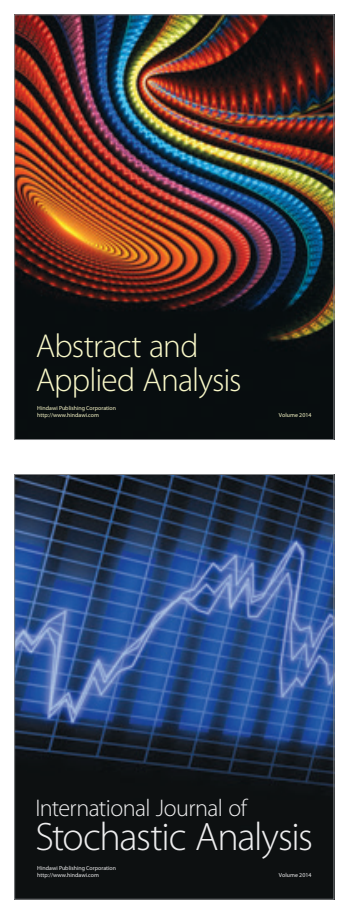

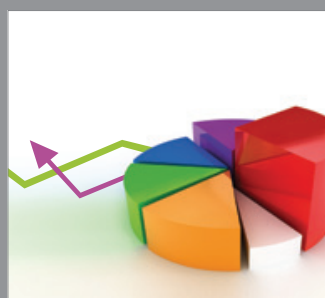

ournal of

Probability and Statistics

Promensencen
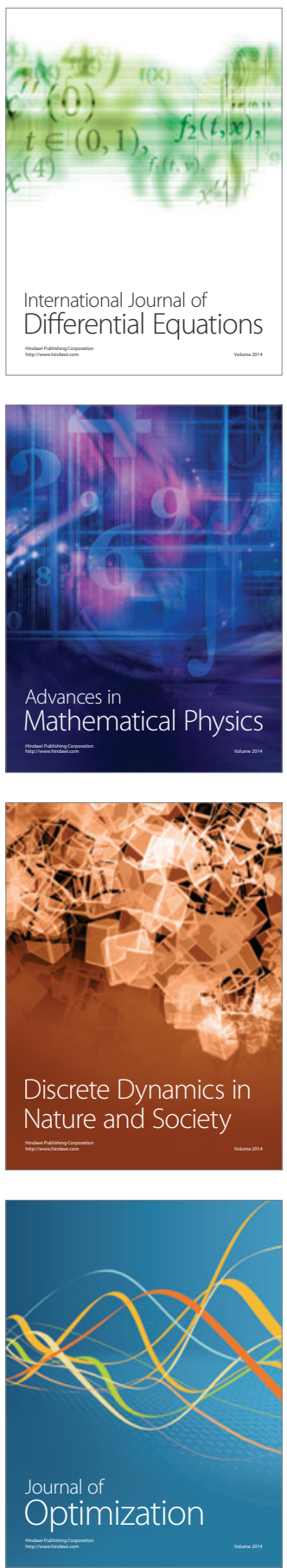